\title{
Empathy at the Gates: Reassessing Its Role in Moral Decision Making
}

\author{
Afreen S. Khalid ${ }^{1 *}$ and Stephan Dickert ${ }^{1,2}$ \\ ${ }^{1}$ Department of Cognitive Psychology, Institute of Psychology, University of Klagenfurt, Klagenfurt, Austria, ${ }^{2}$ Department of \\ Marketing, School of Business and Management, Queen Mary University of London, London, United Kingdom
}

Keywords: empathy, compassion, affective empathy, norms, value-based choice, rationality

\section{INTRODUCTION}

A plethora of research conducted in the past decades has shown that empathy can be essential in guiding and motivating prosocial behavior (e.g., Batson et al., 1981; Eisenberg and Miller, 1987; Batson, 2011; Dickert et al., 2011; Erlandsson et al., 2015). However, there is still considerable debate around whether empathy-driven altruism does more harm than good. For example, several parochial biases have been linked to empathy (Bloom, 2016), such as a preference for helping ingroup over out-group members (Cikara and Fiske, 2012) and identifiable over statistical victims (Small and Loewenstein, 2003; Kogut and Ritov, 2005). In response to the limited and scopeinsensitive empathic responses to large numbers of victims (Slovic, 2007; Västfjäll et al., 2014), suggestions have been put forward that moral decisions should be guided by rational compassion rather than empathy (Bloom, 2016).

In this article, we attempt to clarify some main points of confusion in the discourse surrounding

OPEN ACCESS

Edited by:

Conny Ernst-Peter Wollbrant, University of Stirling, United Kingdom

Reviewed by:

Daniel Vastfjall,

Linköping University, Sweden Andrea Martinangeli,

Burgundy School of Business, France

*Correspondence:

Afreen S. Khalid

afreenksm@gmail.com

Specialty section: This article was submitted to

Cognition,

a section of the journal

Frontiers in Psychology

Received: 24 October 2021

Accepted: 04 January 2022

Published: 27 January 2022

Citation:

Khalid AS and Dickert S (2022) Empathy at the Gates: Reassessing Its Role in Moral Decision Making. Front. Psychol. 13:800752 doi: $10.3389 /$ fpsyg.2022.800752 the merits and pitfalls of empathy. In doing so, we also lay out several possible directions for future research.

\section{DEFINING EMPATHY}

Much of the disagreement surrounding the utility of empathy can be attributed to the nonoverlapping definitions of empathy used in the field. The fuzzy definitions of empathy and compassion have also been pointed out by several different researchers (Neumann et al., 2015; Cuff et al., 2016; Västfjäll et al., 2017; Eklund and Meranius, 2021; Scheffer et al., 2021). Indeed, the lack of a consistent definition of empathy and compassion is a crucial issue which holds back progress in this area of research.

Most commonly, empathy is described as a multi-factorial construct, with (1) an affective component (experience-sharing), which involves feeling the emotions of another person, (2) a cognitive component (perspective-taking/mentalizing), which involves perceiving another person's thoughts or feelings, and (3) a motivational component (compassion/empathic concern), which involves an emotional response that creates the urge to foster the well-being of others (Batson et al., 1997; Decety and Cowell, 2014; Zaki, 2014; Marsh, 2018).

While empathy is described as having several distinct components, critics of empathy (Prinz, 2011; Singer and Klimecki, 2014; DeSteno, 2015; Bloom, 2017) often equate it exclusively to its affective component and consider compassion a distinct process with the capacity to motivate prosocial behavior in more effective ways than empathy (Scheffer et al., 2021). On the other hand, compassion is considered a sub-facet of empathy by many researchers (Decety and Cowell, 2014). Whether compassion should be classified as a sub-component of empathy or if it is a separate process is still an open question which future research should examine. Recent research has already begun investigating the different components of empathy and whether they are separate or co-occur in 
daily life experiences (for review see Weisz and Cikara, 2020; Depow et al., 2021). One possibility laid out by Decety (2021), based on an understanding of the evolutionary roots of empathy, is that empathy's core constituents are (1) emotion sharing, which evolved to facilitate cooperation, and (2) concern for others' well-being, which is an adaptive mechanism that evolved to facilitate the care of offspring. According to this multidisciplinary perspective, these core components are influenced by elements such as perspective-taking and theory of mind. While this is a promising theoretical framework, in light of the definitional challenge of empathy, more research is needed to examine what components constitute empathy, to what degree and in which contexts these components co-activate, and how these components differentially (or collectively) facilitate prosocial behavior.

\section{EMPATHY AS A VALUE-BASED CHOICE}

Empathy is often described both as an automatic, intractable reaction and as a limited resource that can be depleted with overuse (Decety and Cowell, 2014; Bloom, 2016). These limitations of empathy have been cited as a reason behind its parochialism and insensitivity to statistical victims. However, alternative accounts of empathy have been put forward (Zaki, 2014; Cameron et al., 2017) which argue that, instead of being only intuitive and uncontrollable, empathy can be a motivated phenomenon which individuals choose to approach or avoid based on perceived costs and benefits. The malleability of empathy implies that suggestions to discard empathy as a poor moral compass may be premature because empathy can be shaped toward more positive outcomes.

In support of this view, research that looks at the cognitive effort costs of empathizing suggests that empathy's biases may not be inherent in empathy itself but caused by shifts in incentives. When people view empathy as hard work, they might choose to avoid it if given the opportunity (Cameron et al., 2019). People are also more willing to bear the costs of empathy when they have more incentive to do so, such as when it involves members of their kin. This is in line with previous research showing that people avoid empathy in situations where the costs of helping would be too high (Shaw et al., 1994).

One limitation of these studies is that most of them either used a broad definition of empathy or just investigated affective empathy. Whether different components of empathy are affected differentially by motivational cues is still an important question for future research (Ferguson et al., 2020). Another crucial point to note is that while critics of empathy usually recognize that empathy is subject to motivational biases, they fail to make the same judgment for compassion. However, results of a recent study support the view that compassion suffers from some of the same biases as empathy, perhaps even to a greater degree (Scheffer et al., 2021). This study indicated that participants perceived compassion as more cognitively costly than empathy, especially when applied to strangers, which is at odds with the view of compassion as less exhausting than empathy and more likely to lead to sustained helping (Bloom, 2017). Indeed, scope insensitivity has been observed for a range of emotions, including empathy, sympathy, and compassion (Kogut and Ritov, 2005; Dickert and Slovic, 2009; Cameron and Payne, 2011; Västfjäll et al., 2014).

Some of the above research conceptualizes empathy as a value-based choice. From a value-based perspective, individuals make a choice based on its relative subjective value. Thus, under this framework, people might choose to feel empathy by (un)consciously considering its costs (effort, time, money) and benefits (monetary rewards, norm conformity, status). Indeed, research in moral decision-making has begun to make the case for a value-based framework which can help reconcile conflicting findings in the literature (Cameron et al., 2017; Pärnamets et al., 2020).

While more work is needed to understand whether (or when) empathy is best conceptualized as a value-based choice or if it is, as often suggested, an automatic, intuitive response unaffected by value processes, empathy can be a dynamic system that shifts with changing values. Consequently, if the aim is to expand the circle of individuals for whom we feel empathy (Singer, 1981), then changing underlying motives or incentives might be a useful direction toward this.

\section{AFFECTIVE EMPATHY: NOT ALL BAD}

Affective empathy is subject to a number of biases. We can be insensitive to the number of those suffering (Slovic, 2007; Västfjäll et al., 2014; Dickert et al., 2015), biased toward in-group members (Harris and Fiske, 2006), and prefer helping identifiable victims over faceless masses (Small et al., 2007; Lee and Feeley, 2016). Our internal "empathy meters" often don't scale up with the magnitude of the problem.

However, this aspect does not necessitate the dismissal of empathy as a whole. For one, empathy is an evolved mechanism that serves adaptive functions when it comes to caring for the young and coordinating toward achieving a shared goal (Preston and de Waal, 2002; Cohen et al., 2006; Preston, 2013; de Waal and Preston, 2017). It is possible that intuitive, affective empathy constitutes a primary mechanism for helping in the first place (Decety, 2021). Thus, it could very well serve as an activation process by which further cost and benefit calculations are triggered.

Moreover, some evidence suggests that empathy has expanded to a wider circle of individuals over the past few decades (Pinker, 2011). The increasing efforts toward globalization in several domains of life such as economics, culture, politics and communication as well as technological innovations may create the initial sparks of empathy that allow people to consider the perspective of those outside their immediate in-group (Bhagwati and MacMillan, 2004; Pinker, 2011). These, in-turn, may pave the ways for policies and norms protecting the rights of minority groups, which have already been embedded into the moral psychology of some cultures. Ultimately, these policies and norms may affect our experience of empathy. Indeed, this is consistent with research on how incentives and culture affect the empathic experience (Atkins 
et al., 2016; Nook et al., 2016). This suggests that increasing opportunities for intuitive empathy combined with changing existing norms and incentives will be a key aspect of expanding our circle of empathy. In summary, affective empathy is an instinctive, evolved phenomenon which is important for social functioning. While it would appear that it is often intuitive and automatic, the history of moral progress and evidence from contemporary research in psychology suggests that empathy may reflect values which are, at least in part, changeable.

\section{CONCLUSION: MOTIVATED BY THE HEART, GUIDED BY THE HEAD}

The research summarized here suggests several ways for empathy research to move forward. First, it is important to figure out exactly which sub-components fall under the empathy umbrella. How these sub-components operate independently and in concert is another crucial question for future research. Future research should also test whether interventions influence all components of empathy, or whether they are more effective for certain components over others. The efficacy of different motivational cues (such as financial rewards, social rewards or psychological benefits like warm glow) should also be tested on each empathic sub-component.

Further, while claims have been made by researchers on how our circle of empathy has expanded over the centuries, causal research on how our morals change is limited (Bloom, 2010; Andreoni et al., 2021). There is some research which suggests that decision framing matters for aiming to expand our circle of moral regard (Laham, 2009). However, our understanding of this process would benefit from longitudinal research on what processes trigger changes in norms and

\section{REFERENCES}

Andreoni, J., Nikiforakis, N., and Siegenthaler, S. (2021). Predicting social tipping and norm change in controlled experiments. Proc. Natl. Acad. Sci. U.S.A. 118:e2014893118. doi: 10.1073/pnas.2014893118

Atkins, D., Uskul, A. K., and Cooper, N. R. (2016). Culture shapes empathic responses to physical and social pain. Emotion 16, 587-601. doi: $10.1037 / \mathrm{emo} 0000162$

Batson, C. D. (2011). "Empathy-induced altruism: friend or foe of the common good?" in For the Greater Good of All, eds D. R. Forsyth and C. L. Hoyt (New York, NY: Palgrave Macmillan). doi: 10.1057/9780230116269_3

Batson, C. D., Duncan, B. D., Ackerman, P., Buckley, T., and Birch, K. (1981). Is empathic emotion a source of altruistic motivation? J. Pers. Soc. Psychol. 40:290. doi: 10.1037/0022-3514.40.2.290

Batson, C. D., Early, S., and Salvarani, G. (1997). Perspective taking: Imagining how another feels versus imaging how you would feel. Pers. Soc. Psychol. Bull. 23, 751-758. doi: 10.1177/0146167297237008

Bhagwati, J., and MacMillan, M. (2004). In Praise of Globalization. Oxford: Oxford University Press.

Bicchieri, C., and Funcke, A. (2018). Norm Change: Trendsetters and Social Structure. Soc. Res. Int. Quart. 85, 1-21. Available online at: https://www.muse. jhu.edu/article/692742

Bloom, P. (2010). How do morals change? Nature 464:490. doi: 10.1038/464490a emotions of individuals and what the causal chain of this process is.

On a related note, some scholars have also argued that initiators of norm abandonment (i.e., trendsetters) are a crucial part of the norm change process (Bicchieri and Funcke, 2018). Effective Altruism (EA)-a movement based on using evidence and reason to do the most good-attempts to initiate such a change (Caviola et al., 2021). The central message espoused by its proponents is that individuals in affluent countries are morally obligated to donate to socially distant individuals living in extreme poverty (Singer, 1972, 2015). Focusing on aspects such as effectiveness and efficacy also allows comparisons and makes help more quantifiable. Recent research suggests that donors do not instinctively consider the efficacy of their donations (Burum et al., 2020). Making efficacy salient could shift moral norms and hence make people more sensitive to it.

While the emphasis on effectiveness, efficacy, and rationality may seem like a blow for empathy, this need not be the case. Affective processes might be necessary to create the initial spark that lights the fire of moral progress, as affect-rich stimuli often motivate prosocial behavior (Small and Loewenstein, 2003; Kogut and Ritov, 2005; Erlandsson et al., 2015; Dickert et al., 2016). While empathy and/or compassion are the fuel that kick starts our morality, tools such as logic, critical thinking, utilitarian cost-benefit analyses, argumentation with others and reasoning based on empiricism can serve as the steering wheel-allowing us to recognize and reach our preferred destination (Decety, 2021; Pinker, 2021) and perhaps shift our very experience of empathy.

\section{AUTHOR CONTRIBUTIONS}

$\mathrm{AK}$ and SD contributed to writing and editing the manuscript. Both authors contributed to the article and approved the submitted version.

Bloom, P. (2016). Against Empathy: The Case for Rational Compassion. New York, NY: Harper Collins.

Bloom, P. (2017). Empathy and its discontents. Trends Cogn. Sci. 21, 24-31. doi: 10.1016/j.tics.2016.11.004

Burum, B., Nowak, M. A., and Hoffman, M. (2020). An evolutionary explanation for ineffective altruism. Nat. Human Behav. 4, 1245-1257. doi: 10.1038/s41562-020-00950-4

Cameron, C. D., Hutcherson, C. A., Ferguson, A. M., Scheffer, J. A., Hadjiandreou, E., and Inzlicht, M. (2019). Empathy is hard work: People choose to avoid empathy because of its cognitive costs. J. Experi. Psychol. 148:962. doi: 10.1037/xge0000595

Cameron, C. D., and Payne, B. K. (2011). Escaping affect: How motivated emotion regulation creates insensitivity to mass suffering. J. Pers. Soc. Psychol. 100, 1-15. doi: 10.1037/a002 1643

Cameron, D., Cunningham, W., Saunders, B., and Inzlicht, M. (2017). The ends of empathy: Constructing empathy from value-based choice. Trends Cogn. Sci. 25, 213-227. doi: 10.31234/osf.io/d99bp

Caviola, L., Schubert, S., and Greene, J. D. (2021). The psychology of (In) effective altruism. Trends Cogn. Sci. 25, 596-607. doi: 10.1016/j.tics.2021.03.015

Cikara, M., and Fiske, S. T. (2012). Stereotypes and schadenfreude: Affective and physiological markers of pleasure at outgroup misfortunes. Soc. Psychol. Personal. Sci. 3, 63-71. doi: 10.1177/1948550611409245 
Cohen, T. R., Montoya, R. M., and Insko, C. A. (2006). Group morality and intergroup relations: Cross-cultural and experimental evidence. Pers. Soc. Psychol. Bull. 32, 1559-1572. doi: 10.1177/0146167206291673

Cuff, B. M. P., Brown, S. J., Taylor, L., and Howat, D. J. (2016). Empathy: A review of the concept. Emot. Rev. 8, 144-153. doi: 10.1177/1754073914558466

de Waal, F. B., and Preston, S. D. (2017). Mammalian empathy: behavioural manifestations and neural basis. Nat. Rev. Neurosci. 18, 498-509. doi: $10.1038 / \mathrm{nrn} .2017 .72$

Decety, J. (2021). Why empathy is not a reliable source of information in moral decision making. Curr. Dir. Psychol. Sci. 30, 425-430. doi: $10.1177 / 09637214211031943$

Decety, J., and Cowell, J. M. (2014). The complex relation between morality and empathy. Trends Cogn. Sci. 18, 337-339. doi: 10.1016/j.tics.2014.04.008

Depow, G. J., Francis, Z., and Inzlicht, M. (2021). The experience of empathy in everyday life. Psychol. Sci. 32, 1198-1213. doi: 10.1177/0956797621995202

DeSteno, D. (2015). Compassion and altruism: how our minds determine who is worthy of help. Curr. Opin. Behav. Sci. 3, 80-83. doi: 10.1016/j.cobeha.2015.02.002

Dickert, S., Kleber, J., Västfjäll, D., and Slovic, P. (2016). Mental imagery, impact, and affect: A mediation model for charitable giving. PLoS ONE 11:e0148274. doi: 10.1371/journal.pone.0148274

Dickert, S., Sagara, N., and Slovic, P. (2011). Affective motivations to help others: A two-stage model of donation decisions. J. Behav. Decis. Mak. 24, 361-376. doi: 10.1002/bdm.697

Dickert, S., and Slovic, P. (2009). Attentional mechanism in the generation of sympathy. Judgm. Decis. Mak. 4, 297-306.

Dickert, S., Västfjäll, D., Kleber, J., and Slovic, P. (2015). Scope insensitivity: The limits of intuitive valuation of human lives in public policy. J. Appl. Res. Mem. Cogn. 4, 248-255. doi: 10.1016/j.jarmac.2014.09.002

Eisenberg, N., and Miller, P. A. (1987). The relation of empathy to prosocial and related behaviors. Psychol. Bull. 101, 91-119. doi: 10.1037/0033-2909.101.1.91

Eklund, J. H., and Meranius, M. S. (2021). Toward a consensus on the nature of empathy: A review of reviews. Patient Educ. Couns. 104, 300-307. doi: 10.1016/j.pec.2020.08.022

Erlandsson, A., Björklund, F., and Bäckström, M. (2015). Emotional reactions, perceived impact and perceived responsibility mediate the identifiable victim effect, proportion dominance effect and in-group effect respectively. Organ. Behav. Hum. Decis. Process. 127, 1-14. doi: 10.1016/j.obhdp.2014.11.003

Ferguson, A. M., Cameron, C. D., and Inzlicht, M. (2020). Motivational effects on empathic choices. J. Exp. Soc. Psychol. 90:104010. doi: 10.1016/j.jesp.2020.104010

Harris, L. T., and Fiske, S. T. (2006). Dehumanizing the lowest of the low: Neuroimaging responses to extreme out-groups. Psychol. Sci. 17, 847-853. doi: $10.1111 / j .1467-9280.2006 .01793 . x$

Kogut, T., and Ritov, I. (2005). The "identified victim" effect: An identified group, or just a single individual?. J. Behav. Decis. Mak. 18, 157-167. doi: $10.1002 / \mathrm{bdm} .492$

Laham, S. M. (2009). Expanding the moral circle: Inclusion and exclusion mindsets and the circle of moral regard. J. Exp. Soc. Psychol. 45, 250-253. doi: 10.1016/j.jesp.2008.08.012

Lee, S., and Feeley, T. H. (2016). The identifiable victim effect: A meta-analytic review. Soc. Influ. 11, 199-215. doi: 10.1080/15534510.2016.1216891

Marsh, A. A. (2018). The neuroscience of empathy. Curr. Opin. Behav. Sci. 19, 110-115. doi: 10.1016/j.cobeha.2017.12.016

Neumann, D. L., Chan, R. C. K., Boyle, G. J., Wang, Y., and Westbury, H. R. (2015). "Measures of empathy: Self-report, behavioral, and neuroscientific approaches," in Measures of Personality and Social Psychological Constructs, eds G. J. Boyle, D. H. Saklofske, and G. Matthews (Elsevier Academic Press). doi: 10.1016/B978-0-12-386915-9.00010-3

Nook, E. C., Ong, D. C., Morelli, S. A., Mitchell, J. P., and Zaki, J. (2016). Prosocial conformity: Prosocial norms generalize across behavior and empathy. Pers. Soc. Psychol. Bull. 42, 1045-1062. doi: 10.1177/014616721664 9932
Pärnamets, P., Shuster, A., Reinero, D. A., and Van Bavel, J. J. (2020). A value-based framework for understanding cooperation. Curr. Dir. Psychol. Sci. 29, 227-234. doi: 10.1177/0963721420906200

Pinker, S. (2011). The Better Angels of Our Nature: Why Violence Has Declined. New York, NY: Viking.

Pinker, S. (2021). Rationality: What it Is, Why it Seems Scarce, Why it Matters. New York, NY: Viking.

Preston, S. D. (2013). The origins of altruism in offspring care. Psychol. Bull. 139, 1305-1341. doi: 10.1037/a0031755

Preston, S. D., and de Waal, F. B. M. (2002). Empathy: Its ultimate and proximate bases. Behav. Brain Sci. 25, 1-20. doi: 10.1017/S0140525X02000018

Prinz, J. (2011). Against empathy. South. J. Philos. 49, 214-233. doi: 10.1111/j.2041-6962.2011.00069.x

Scheffer, J. A., Cameron, C. D., and Inzlicht, M. (2021). Caring is costly: People avoid the cognitive work of compassion. J. Exp. Psychol. Gen. doi: $10.1037 /$ xge0001073

Shaw, L. L., Batson, C. D., and Todd, R. M. (1994). Empathy avoidance: Forestalling feeling for another in order to escape the motivational consequences. J. Pers. Soc. Psychol. 67, 879-887. doi: 10.1037/0022-3514.67. 5.879

Singer, P. (1972). Famine, affluence, and morality. Philos. Public Aff. 1972, 229-243.

Singer, P. (1981). The Expanding Circle. Oxford: Clarendon Press.

Singer, P. (2015). The Most Good You Can Do. London: Yale University Press.

Singer, T., and Klimecki, O. M. (2014). Empathy and compassion. Curr. Biol. 24:54. doi: $10.1016 /$ j.cub.2014.06.054

Slovic, P. (2007). If I look at the mass I will never act: Psychic numbing and genocide. Judgm. Decis. 2, 79-95. doi: 10.1037/e7183320 07-003

Small, D. A., and Loewenstein, G. (2003). Helping a victim or helping the victim: Altruism and identifiability. J. Risk Uncertain. 26, 5-16. doi: 10.1023/A:1022299422219

Small, D. A., Loewenstein, G., and Slovic, P. (2007). Sympathy and callousness: The impact of deliberative thought on donations to identifiable and statistical victims. Organ. Behav. Hum. Decis. Process. 102, 143-153. doi: 10.1016/j.obhdp.2006.01.005

Västfjäll, D., Erlandsson, A., Slovic, P., and Tinghög, G. (2017). Commentary: empathy and its discontents. Front. Psychol. 8:542. doi: 10.3389/fpsyg.2017.00542

Västfjäll, D., Slovic, P., Mayorga, M., and Peters, E. (2014). Compassion fade: Affect and charity are greatest for a single child in need. PLoS ONE 9:e100115. doi: 10.1371/journal.pone.0100115

Weisz, E., and Cikara, M. (2020). Strategic regulation of empathy. Trends Cogn. Sci. doi: $10.31234 /$ osf.io/2kr46

Zaki, J. (2014). Empathy: A motivated account. Psychol. Bull. 140, 1608-1647. doi: $10.1037 / \mathrm{a} 0037679$

Conflict of Interest: The authors declare that the research was conducted in the absence of any commercial or financial relationships that could be construed as a potential conflict of interest.

Publisher's Note: All claims expressed in this article are solely those of the authors and do not necessarily represent those of their affiliated organizations, or those of the publisher, the editors and the reviewers. Any product that may be evaluated in this article, or claim that may be made by its manufacturer, is not guaranteed or endorsed by the publisher.

Copyright (c) 2022 Khalid and Dickert. This is an open-access article distributed under the terms of the Creative Commons Attribution License (CC BY). The use, distribution or reproduction in other forums is permitted, provided the original author(s) and the copyright owner(s) are credited and that the original publication in this journal is cited, in accordance with accepted academic practice. No use, distribution or reproduction is permitted which does not comply with these terms. 